\title{
Erratum to: A Simple Model for the Vertical Transport of Reactive Species in the Convective Atmospheric Boundary Layer
}

\author{
Leif Kristensen - Donald H. Lenschow • \\ David Gurarie • Niels Otto Jensen
}

Published online: 19 February 2010

(C) Springer Science+Business Media B.V. 2010

\section{Erratum to: Boundary-Layer Meteorol DOI 10.1007/s10546-009-9443-x}

We have discovered an error in Eq. 76, in our article Kristensen et al. (2010). The factor $\theta_{4}$ was missing inside the braces in the numerator. Here we present the correct equation.

$$
\gamma(\zeta)=\frac{\theta_{4} \varphi_{T}\left\{\theta_{4} \mathscr{D}\left(F_{2} S_{1}+F_{1} S_{2}\right)-\theta_{1}\left(\omega^{2}+\theta_{4} \varphi_{T}\right) \frac{\partial S}{\partial \zeta}\right\}}{\theta_{1}\left\{1+\mathscr{D}\left(1+S_{1}+S_{2}\right) \theta_{4}\right\}\left\{\omega^{2}+\theta_{4} \varphi_{T}\right\}} .
$$

Unfortunately, this mistake created serious errors in Figs. 2, 3, 4, and 5. The corrected figures are shown below. The bottom frames of Fig. 3 show in more detail the diffusivities close to the surface in linear-logarithmic plots.

Comparison with the old figures shows significant differences in all the figures. The most important observation is that the $\mathrm{O}_{3}$ and $\mathrm{NO}$ diffusivities are very similar and never negative. However, the $\mathrm{NO}_{2}$ diffusivities are considerably modified, in particular in the surface layer. They all have one altitude at which they go to $\pm \infty$. These anomalies are caused by the fact that the $\mathrm{NO}_{2}$ concentration profiles go through a maximum where the derivatives become zero, while the fluxes, which are negative at the surface, have already become greater than zero.

The online version of the original article can be found under doi:10.1007/s10546-009-9443-x.

L. Kristensen $(\varangle) \cdot$ N. O. Jensen

Ris $\varnothing$ DTU National Laboratory for Sustainable Energy, Roskilde, Denmark

e-mail: leif.kristensen@net.telenor.dk

D. H. Lenschow

National Center for Atmospheric Research, Boulder, CO, USA

D. Gurarie

Department of Mathematics, Case Western Reserve University, Cleveland, OH, USA 

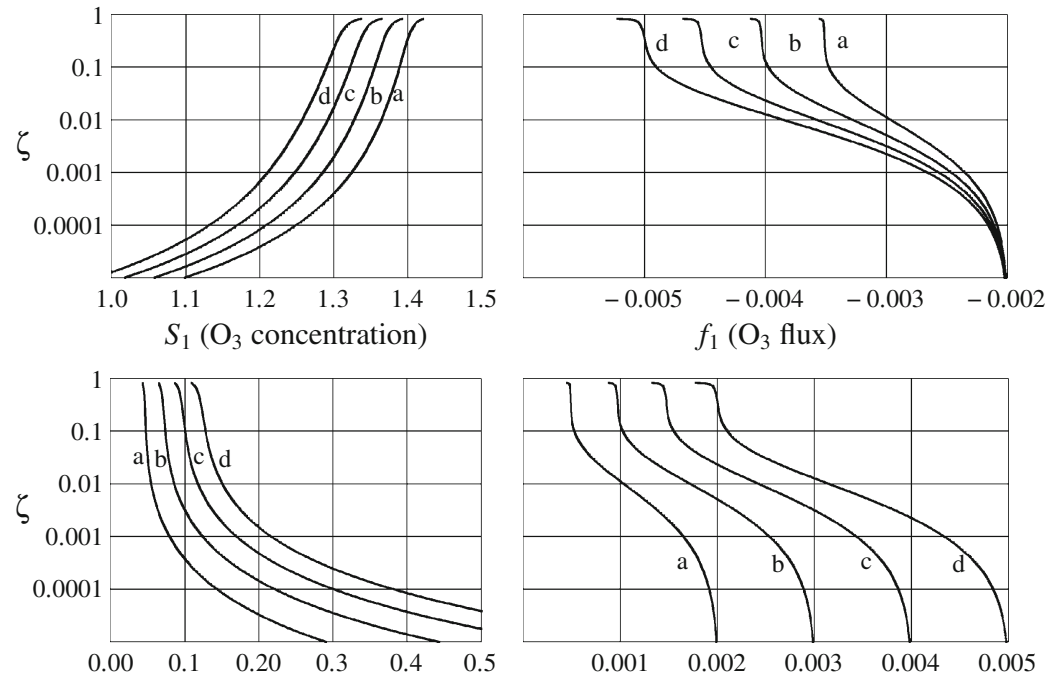

$S_{2}$ (NO concentration)
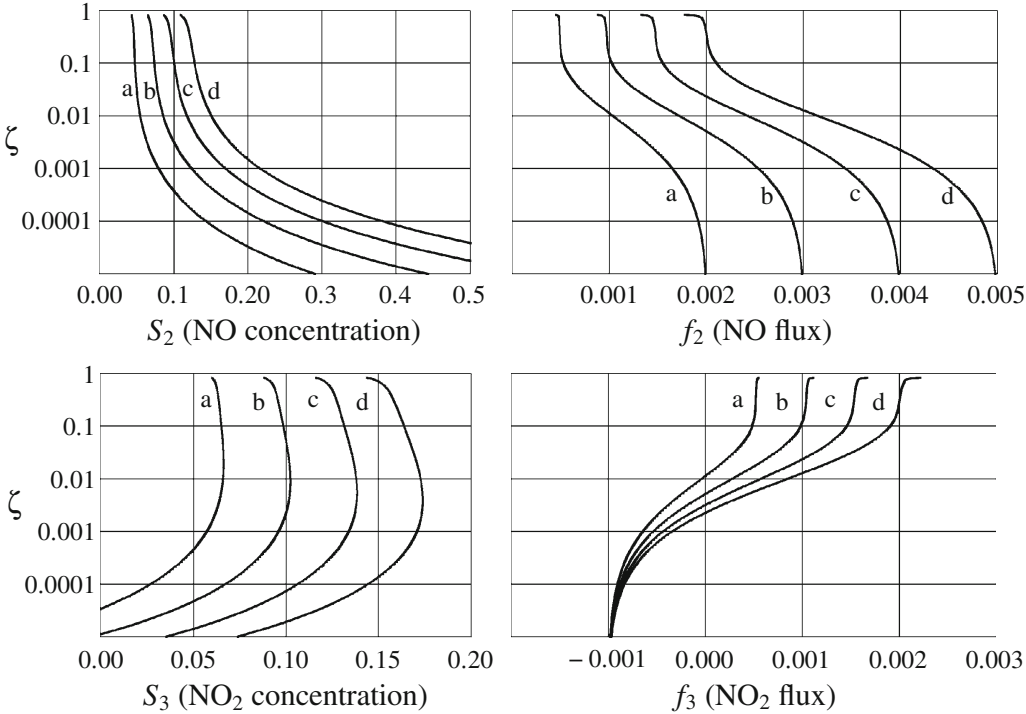

Fig. 2 Profiles of concentrations and fluxes with parameters corresponding to the values given in Table 1 in Kristensen et al. (2010)
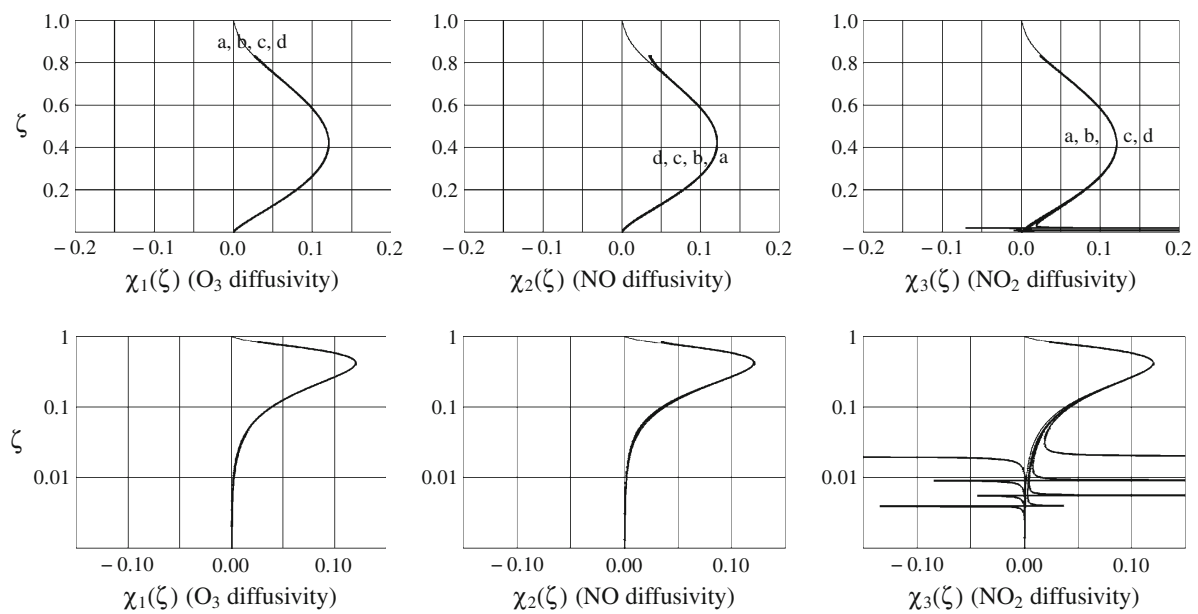

Fig. 3 The dimensionless diffusivity profiles in a linear-linear form (top frames) and in a linear-logarithmic form (bottom frames). In each frame the conserved-species diffusivity is shown with a thin line 

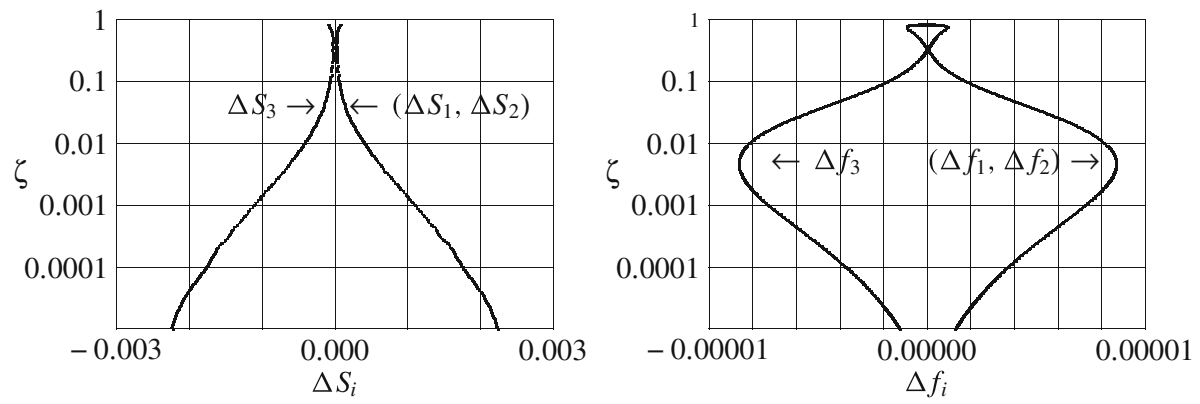

Fig. 4 Profiles of concentration and flux increments $\Delta S_{i}$ and $\Delta f_{i}$ due to the covariance $\left\langle s_{1} s_{2}\right\rangle$ in the case labeled "a"

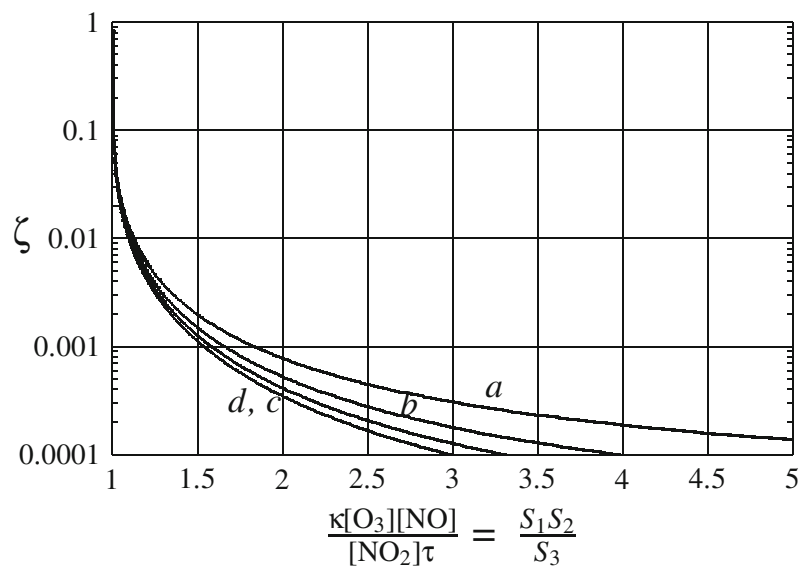

Fig. 5 Profiles of the ratio of production and destruction of $\mathrm{NO}_{2}$. The labels correspond to the four boundary conditions in Table 1 in Kristensen et al. (2010)

\section{Reference}

Kristensen L, Lenschow DH, Gurarie D, Jensen NO (2010) A simple model for the vertical transport of reactive species in the convective atmospheric boundary layer. Boundary-Layer Meteorol 134: 195221. doi:10.1007/s10546-009-9443-x 